\title{
Indoor Fire Localisation using WSN and Fire Map Generation for Industrial Environment
}

\author{
Atharva R Karpate ${ }^{1^{*}}$, Piyush P Agarwal ${ }^{2 *}$ and Ashlesh A Jaiswal ${ }^{3}$ \\ ${ }^{1}$ Department of Electronics and Communication, \\ Shri Ramdeobaba College of Engineering and Management, Nagpur, India
}

\section{ABSTRACT}

Every year Industrial fires lead to tremendous economic and human loss. Real-time fire detection and localisation remains a serious challenge, especially for the industrial environment. With the substantial growth of Wireless Sensor Network(WSN) in recent years, one of the most important applications of it has been in the area of fire detection and protection. In this paper, we present a low-cost and scalable wireless sensor network-based system for fire detection and localisation. To analyse the fire source and spread in real-time we also present a fire map generation mechanism in our system. Various sensor configurations are tried to optimize fire detection and localisation coverage.

\section{KEY WORDS: WIRELESS SENSOR NETWORK, FIRE DETECTION, INDOOR LOCALIZATION, FIRE MAP GENERATION.}

\section{INTRODUCTION}

Fire detection and prevention systems constitute a crucial part of any industrial establishment. In recent years, there has been an increase in the number of fire-related accidents which have resulted in significant loss of lives and properties. An efficient and effective system can reduce losses considerably. Consequently, much research has been carried out in the area of fire detection and prevention. One such example is the use of wireless sensor networks for fire detection. A Wireless Sensor Network(WSN) is a collection of multiple sensor nodes which communicate with a set of gateways to transfer information about their environment. Wireless systems have an advantage in emergency fire situations as they are less prone to damages caused by the fire source and are easy to install compared to wired systems. Wireless

\section{ARTICLE INFORMATION}

*Corresponding Author: karpatear@rknec.edu

Received 17th Oct 2020 Accepted after revision 29th Dec 2020

Print ISSN: 0974-6455 Online ISSN: 2321-4007 CODEN: BBRCBA

Thomson Reuters ISI Web of Science Clarivate Analytics USA and Crossref Indexed Journal

\section{Clarivate
Analytics}

NAAS Journal Score 2020 (4.31)

A Society of Science and Nature Publication,

Bhopal India 2020. All rights reserved.

Online Contents Available at: http//www.bbrc.in/

Doi: http://dx.doi.org/10.21786/bbrc/13.14/97 sensor nodes also have other advantages such as low cost, flexibility in the operating environment and low power consumption per node.

Literature Review: Traditionally, fire detection systems have relied on smoke sensors to warn for possible fires. These systems generally do not have monitoring devices and do not help with localising fire. Efforts have been put to design WSN based fire detection systems, [1] combines smoke and temperature sensors to detect fire and reduce false positives. [2]To ensure the safety of workers working in mines, various network topologies and communication protocols were analyzed for the optimum performance of a WSN-based fire detection system. Apart from mere fire detection, [3] uses multiple sensors on a wireless node with trilateration technique to localise fire in the indoor environment. [4] A framework is designed to detect fire and simultaneously perform rescue operations with the help of WSN based technology.

The framework consists of a fire detection sensor network, middleware, data gathering layer, and an escape system. Vision sensors or a combination of vision and smoke sensors have been used in many fire detection systems $[5,6]$. Though vision sensors help in getting real-time visual information, these systems have high deployment costs and are less effective in large indoor environments

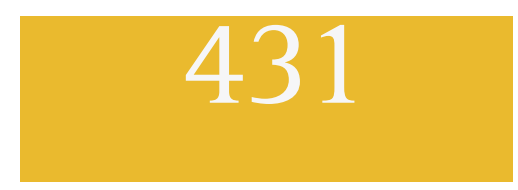


which are typical of industrial settings. In this paper, we present a low cost and scalable system for fire detection and localisation using WSN. The paper also presents an analysis of various sensor configurations in each wireless node. Finally, the paper proposes the generation of a fire map in real-time to help with the monitoring and mitigation of fire sources. The paper is divided into three sections starting with the overview of the proposed system followed by its detailed architecture and analysis.

\section{Overview of the System.}

Figure 1: Overview of the System

Fire Detection $\Rightarrow($ Fire Localization $) \rightrightarrows\left(\begin{array}{l}\text { Fire Map } \\ \text { Generation }\end{array}\right)$

The proposed system is divided into three parts as follows -

1. Fire Detection subsystem - Fire detection subsystem has a high sensitivity for smoke and is primarily tasked with detecting fire. This system is continuously polling for signs of smoke and fire.

2. Fire Localisation subsystem - After the successful detection of fire, it is important to localise the position of fire. This subsystem is tasked with localising the fire source and it's spread.

3. Fire Map Generation subsystem - Once the fire is localised, this subsystem acts to produce real-time Fire Map of the environment which tracks the source and spread of fire.

Figure 2: Block Diagram of Wireless Node

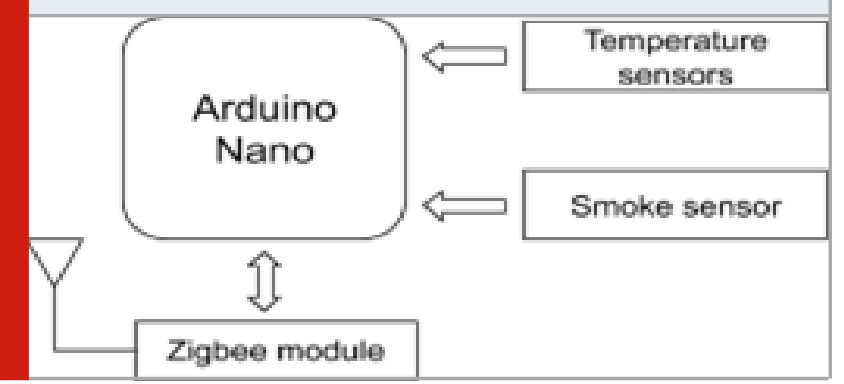

Hardware Description and System Architecture: We propose a simple wireless node for the system which is a low cost, low power consumption and scalable wireless sensor node as shown in Figure 2. The Node consists of a simple microcontroller-Arduino Nano, Zig-Bee module for wireless communication, Smoke sensor and IR temperature sensors for detecting and localising fire respectively.

\section{Hardware Components}

1. Arduino Nano - Arduino Nano is an open-source embedded computing platform based on Atmega 328 single-chip microcontroller. Arduino provides a development environment which utilizes Processing for creating stand-alone embedded systems applications. We use this as the primary processing unit in the Wireless Node.

2. Zig - Bee module - Zig- Bee is a specification for a group of high-level communication protocols targeted at low power, low data rate and low-cost wireless devices. It has a range of 100-120 meters and has a defined bandwidth of $250 \mathrm{~kb} / \mathrm{s}$. The Zig-Bee module acts as the communication medium between each node and the central node in our system.

3. Smoke sensor - MQ-2 Gas sensor is useful for detecting $\mathrm{H} 2$, LPG, $\mathrm{CH} 4$, CO, Alcohol and smoke. The sensor provides digital as well as analog outputs for measurement with a range of 200 to 10,000 PPM. Smoke sensors have high sensitivity but do not help with the localisation of fire. As smoke is pervasive in nature, smoke arising from one area could easily be detected in other areas. Hence, we have used the MQ-2 Smoke sensor primarily for the Fire Detection subsystem.

4. IR Temperature Sensor - We use IR temperature sensors in combination with the MQ-2 Gas sensor in order to localise the fire source. For the proposed system, we have used MLX90614ESF-AAA IR Temperature sensor. It is a high-resolution and highaccuracy non-contact temperature sensor with a 90-degree field of view and can detect temperatures between -70 to $380^{\circ} \mathrm{C}$. This sensor uses a digital interface(SMBus) and can provide continuous readings with customizable 10-bit PWM output.

Figure 3: Workflow of Detection and Localisation subsystems.

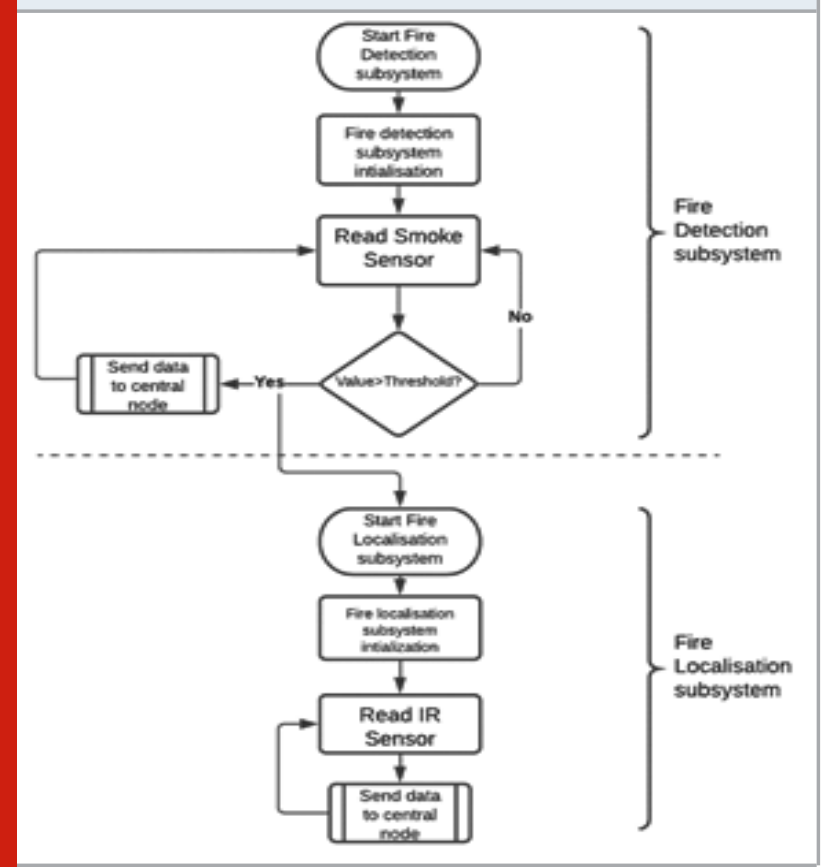

\section{System Architecture}

Fire Detection Subsystem: Fire detection is the first step in the proposed system. MQ - 2 gas sensors are used to 
detect smoke from the fire source. Each MQ - 2 Smoke sensor is equipped with multiple IR Temperature sensors for fire source and spread localisation. As MQ - 2 smoke sensors have low specificity we do not use them for fire localisation. Once the PPM of smoke crosses a preset threshold value, the detection subsystem activates the fire localisation subsystem and sends this data to the central node for the fire map generation.

Fire Localisation Subsystem: After the successful detection of fire, it is important to localise the fire in real-time. To localise the fire source and its spread, this subsystem uses multiple IR temperature sensors(MLX90614ESF-AAA). This subsystem is not activated until the fire is detected by the fire detection subsystem. This saves power as only one sensor is polling continuously in each wireless node. After fire detection, each IR temperature sensor detects the temperature within its surrounding and this data is reported to the central node for the generation of a fire map.

Wireless Pod Design: Each wireless node is termed as a pod. It consists of a smoke sensor at its centre and a varying number of IR Temperature sensors. The pod is further divided into cells based on the location of IR Temperature sensors. These cells are arranged in different patterns to get multiple Pod configurations. To detect and localise the fire source in each WSN Node or Pod, we propose various Pod Configurations based on the following parameters -

1. Percentage of Detection Area - This is defined as the percent of area out of the total area within a Pod which can timely detect the presence of fire and the concentration of smoke.

2. Percentage of Localisation Area - It is defined as the percent of area out of the total area within a Pod where a fire source can be accurately and timely localised.

3. Number of Sensors - Number and type of sensors(Smoke and IR Temperature Sensor) required per pod.

4. Pod Shape Scalability - The Pod design should have a repeatable geometry so that it could be deployed across various industrial spaces.

5. Taking into consideration the above parameters, we propose the following three configurations -

6. Pod Configuration A - This configuration consists of 4 IR Temperature and 1 Smoke sensors which are positioned as shown in Figure 4. The shape of the pod is square which is inscribed in a circle whose radius is the detection radius of the smoke sensor. This square is further divided into 4 smaller cells and one IR sensor is placed at the centre of each square.

7. Pod Configuration B - This configuration consists of 5 IR Temperature and 1 Smoke sensors which are placed as shown in Figure 4. The shape of the Pod is square whose sides are equivalent to the diameter of the smoke sensor detection circle i.e the detection region is inscribed in the square Pod, which primarily differentiates it from configuration A.
8. Pod Configuration C - This configuration consists of 7 IR Temperature sensors and 1 Smoke sensor placed as shown in Figure 4. The pod consists of seven hexagons, each with 1 IR Temperature sensor at its centre. These seven hexagons are inscribed inside the smoke detection region. These configurations are repeated over the specified industrial environment for detection and localisation coverage.

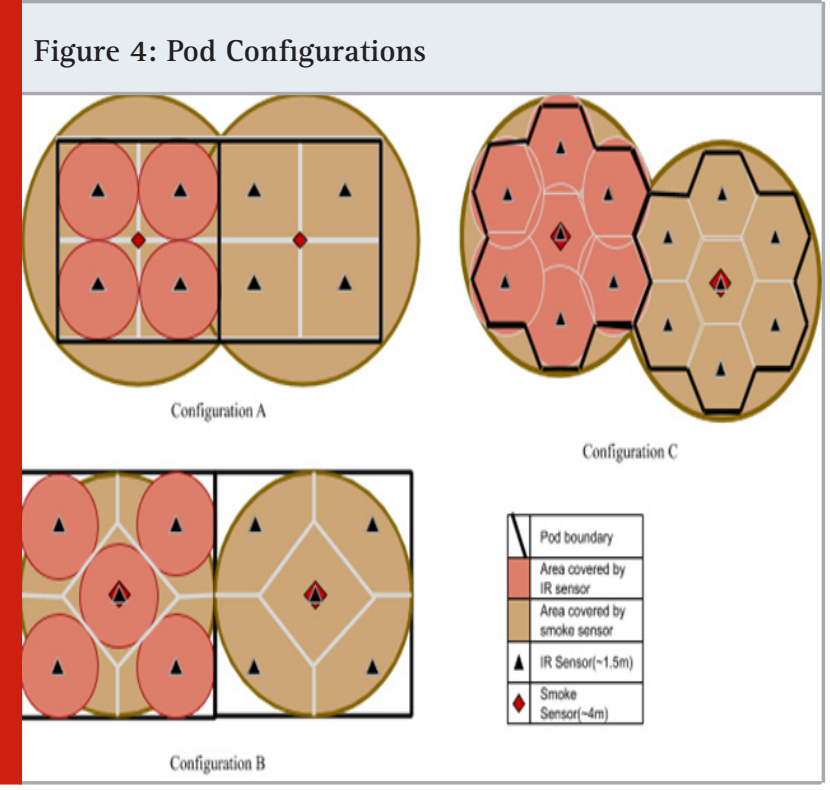

Figure 5: Fire Map

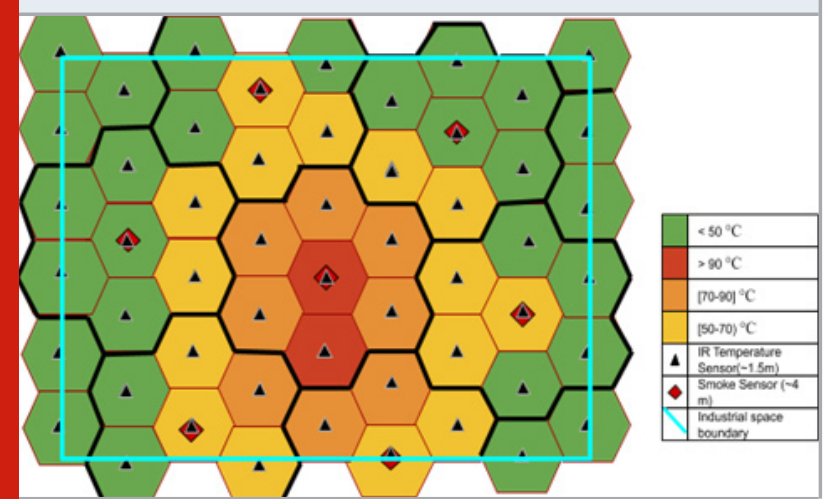

Fire Map Generation: All the wireless nodes are connected to a central node in a star network topology. The central node acts as a hub to communicate and collect information from all nodes. To successfully monitor and localise the fire source, a fire map is generated at the central node. After the successful detection of fire and subsequent collection of localisation information from each pod, a fire map is created as shown in Figure 5. Areas with a high-temperature range of above $90{ }^{\circ} \mathrm{C}$ are classified by red and are considered dangerous. Orange $\left(70-90^{\circ} \mathrm{C}\right)$ and Yellow $\left(50-70^{\circ} \mathrm{C}\right)$ represent milder temperature ranges which are deleterious after long exposure. The green areas represent cells which have room temperature and are completely safe. 
Results and Analysis: Range of Smoke sensor - To find the optimum range of smoke detection for MQ - 2 sensor, readings were collected at different ranges from the fire source as shown in Figure 6. It was observed that the MQ -2 gas sensor provides reliable readings until the distance of $\sim 4$ meters from the fire source. We chose this as the range of smoke sensors and derived our pod configurations from it. Range of IR Temperature sensor - To localise and get the accurate temperature readings, we employ IR Temperature sensors within their optimum detection ranges. To find the feasible radius of temperature detection with an admissible error range, we measured the temperature at different lengths from the fire source and compared it with the temperature near the fire source(thermometer placed at $10 \mathrm{~cm}$ ) as evident in Table 1. The IR Temperature sensor does not provide accurate readings for distances more than $\sim 1.5 \mathrm{~m}$. To keep the system accurate we use this as the optimum range of the IR Temperature sensor.

Figure 6: Range of Smoke Sensor

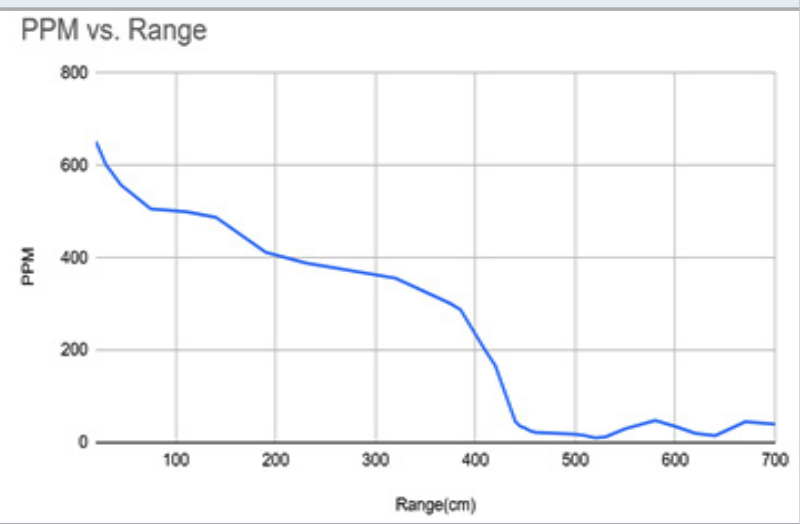

Table 1. Range of IR Temperature Sensor

\begin{tabular}{|l|l|l|l|}
\hline $\begin{array}{l}\text { Distance from } \\
\text { the fire source } \\
(\mathrm{m})\end{array}$ & $\begin{array}{l}\text { Temperature } \\
\text { Sensor }\left({ }^{\circ} \mathrm{C}\right)\end{array}$ & $\begin{array}{l}\text { Thermometer } \\
\left({ }^{\circ} \mathrm{C}\right) \\
\text { At } 10 \mathrm{~cm} \text { from } \\
\text { Fire Source }\end{array}$ & Error \\
\hline 0.1 & 72 & 73 & -1 \\
\hline 0.3 & 74 & 73 & +1 \\
\hline 0.5 & 72 & 74 & -2 \\
\hline 0.7 & 70 & 73 & -3 \\
\hline 1.0 & 71.5 & 74 & -2.5 \\
\hline 1.3 & 71 & 75 & 4 \\
\hline 1.5 & 71.5 & 77 & 5.5 \\
\hline 1.7 & 68.5 & 76 & 7.5 \\
\hline
\end{tabular}

Configuration Results and Explanation: We provide sample analysis for the three configurations based on the
Table 2. Pod configuration analysis

\begin{tabular}{|l|l|l|l|l|l|l|l|l|}
\hline Config & $\begin{array}{l}\text { Area of } \\
\left.\text { Cell( } \mathrm{m}^{2}\right)\end{array}$ & $\begin{array}{l}\text { No. of } \\
\text { cells } \\
\text { in Pod }\end{array}$ & $\begin{array}{l}\text { Area } \\
\text { of Pod } \\
\left(\mathrm{m}^{2}\right)\end{array}$ & $\begin{array}{l}\text { No. of } \\
\text { IR }\end{array}$ & $\begin{array}{l}\text { No. of Smoke } \\
\text { Sensor }\end{array}$ & $\begin{array}{l}\text { Total } \\
\text { Sensor }\end{array}$ & $\begin{array}{l}\text { Detection } \\
\%\end{array}$ & Localisation \% \\
\hline A & 8 & 4 & 32 & 50 & 13 & 63 & 100 & 87.5 \\
\hline B & 16 & 5 & 64 & 31 & 7 & 38 & 78.5 & 55.1 \\
\hline C & 5.8 & 7 & 41 & 69 & 10 & 79 & 100 & 100 \\
\hline
\end{tabular}

results obtained for the detection range of smoke sensor and localisation radius of IR Temperature sensor -

- Sample Floor Area - 400m2

- Smoke sensor radius $\sim 4 \mathrm{~m}$

- Area of Smoke Circle - $50.26 \mathrm{~m} 2$

- IR Sensor radius $1.5 \mathrm{~m}$

- $\quad$ Area of IR Temperature Sensor - 7.06 m2

Table 2 represents the analysis of the three configurations. Based on the detection and localisation region, we calculate the number of IR Temperature sensors, Smoke sensors and the total number of sensors for each configuration. The percent of detection area and the percent of localisation area out of the total area of the pod were also calculated for the three configurations. It was observed that configuration C provides $100 \%$ detection and localisation coverage for the entire pod area but the number of sensors required is the highest amongst the three configurations. On the other hand, Configuration $B$ requires the least number of sensors but has poor detection and localisation capacity. Configuration A provides full coverage for fire detection and sufficient fire localisation coverage, though the number of sensors required is $\sim 65 \%$ more than Configuration B. Configuration $\mathrm{C}$ can be utilized in areas with the need for complete detection and localisation such as nuclear facilities, ordnance factories or any other critical facilities where real-time fire localisation is of foremost importance for fire extinguishment and evacuation effort. Configuration B could be deployed in residential and office premises, where fire detection is the chief factor. Configuration A provides a blend of Configuration B and C, it could be primarily applied to a non-critical industrial environment where complete fire detection and adequate fire localisation are required.

\section{CONCLUSION}

The presented system provides a scalable, low cost and low power fire detection and localisation system. The proposed system generates a fire map to get real-time information about the fire source and its spread. The combination of Smoke and IR Temperature sensors with varying configurations provide a flexible solution for monitoring fire in different industrial environments. Three configurations were analysed in this paper but further improvements could be made by adding multiple sensors and designing configurations according to the need of the industrial environment. A system for targeted fire extinguishment could be added to stop the fire spread in real-time. 


\section{REFERENCES}

Azmil, M.S.A., Ya'Acob, N., Tahar, K.N. and Sarnin, S.S., 2015, March. Wireless fire detection monitoring system for fire and rescue application. In 2015 IEEE 11th International Colloquium on Signal Processing \& Its Applications (CSPA) (pp. 84-89). IEEE.

Deve, K.B., Hancke, G.P. and Silva, B.J., 2016, October. Design of a smart fire detection system. In IECON 2016-42nd Annual Conference of the IEEE Industrial Electronics Society (pp. 6205-6210). IEEE.

Islam, T., Rahman, H.A. and Syrus, M.A., 2015, June. Fire detection system with indoor localization using ZigBee based wireless sensor network. In 2015 international conference on informatics, electronics \& vision (ICIEV) (pp. 1-6). IEEE.
Kanwal, K., Liaquat, A., Mughal, M., Abbasi, A.R. and Aamir, M., 2017. Towards development of a low cost early fire detection system using wireless sensor network and machine vision. Wireless Personal Communications, 95(2), pp.475-489.

Lim, Y.S., Lim, S., Choi, J., Cho, S., Kim, C.K., Hu, Y.W.L.H., Zhang, H., Hu, H., Xu, B., Li, J. and Ma, A., 2007, November. A fire detection and rescue support framework with wireless sensor networks. In 2007 International Conference on Convergence Information Technology (ICCIT 2007) (pp. 135-138). IEEE.

Tan, W., Wang, Q., Huang, H., Guo, Y. and Zhang, G., 2007, July. Mine fire detection system based on wireless sensor network. In 2007 International Conference on Information Acquisition (pp. 148-151). IEEE. 